\title{
ARTICLE OPEN Experimental observation of bulk nodal lines and electronic surface states in $\mathrm{ZrB}_{2}$
}

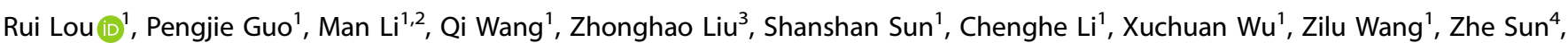
Dawei Shen $\mathbb{i D}^{3}$, Yaobo Huang $\mathbb{D}^{2}$, Kai Liu' ${ }^{1}$, Zhong-Yi Lu${ }^{1}$, Hechang Lei $\mathbb{D}^{1}$, Hong Ding $\mathbb{D}^{5,6,7}$ and Shancai Wang ${ }^{1}$

Topological nodal-line semimetals are characterized by line-contact bulk band crossings and topological surface states. Breaking certain protecting symmetry turns this system into a Dirac semimetal or Weyl semimetal that hosts zero-dimensional isolated nodal points. Recent advances in band theory predicted a topological nodal-line semimetal state possessing a new type of nodal line in $\mathrm{AlB}_{2}$-type diborides. Here, we report an experimental realization of nodal-line fermions and associated surface states near the Fermi energy in $\mathrm{ZrB}_{2}$ by angle-resolved photoemission spectroscopy combined with first-principles calculations. The Dirac nodal lines in $\mathrm{ZrB}_{2}$ wind into two groups of nodal rings, which are linked together along the $\Gamma-K$ direction. We further observe a distinct surface state connecting to each nodal line, indicative of the nontrivial topological nature of the bulk nodal lines. Therefore, our results provide convincing experimental evidence of nodal-line semimetal states in $\mathrm{ZrB}_{2}$ both in the bulk and on the surface, suggesting $\mathrm{ZrB}_{2}$ as a remarkable platform for discovering unique phenomena induced by nodal-line fermions.

npj Quantum Materials (2018)3:43; doi:10.1038/s41535-018-0121-4

\section{INTRODUCTION}

The realization of novel quantum states of matter with nontrivial topology beyond topological insulators has become a significant objective in current condensed-matter physics research. ${ }^{1-3}$ Very recently, the discovery of topological semimetals has achieved this goal, which ignites extensive work focusing on the exotic topological properties and their underlying connection with the electronic structure. ${ }^{4}$ The topological semimetal states host nontrivial bulk band-crossing points in crystal momentum space. ${ }^{5,6}$ Characterized by the degeneracy, distribution of the band-crossing points in the Brillouin zone (BZ), and the associated topological boundary states, the topological semimetals can be classified into three categories: Dirac, Weyl, and nodal-line semimetals. In Dirac and Weyl semimetals, the bulk nodes are discrete in the BZ and their surface projections are connected by surface Fermi arcs. ${ }^{4}$ While in nodal-line semimetals, the bulk nodes extend along one-dimensional curves and the corresponding surface states are flat in dispersion according to the previous nodal-line modelings, ${ }^{7}$ where the band crossings of a nodal line should occur at zero energy with a constraint chiral symmetry. Hence the flat surface bands are dubbed the drumhead states. However, the chiral symmetry is not exact in a real crystal, resulting in the nodal line does not generally occur at a constant energy, thus the associated topological surface states are not flat either. 8,9

The Dirac semimetal and Weyl semimetal states have been theoretically predicted and experimentally verified in various families of compounds. ${ }^{10-21}$ Although there have been several theoretical proposals for the material realization of topological nodal-line semimetal states, ${ }^{4,8,22-24}$ the conclusive experimental proof remains absent until recent angle-resolved photoemission spectroscopy (ARPES) measurements on $\mathrm{TiB}_{2}{ }^{25}$ showing a tangible realization of bulk nodal-line fermions. Since the surface states associated with the nodal lines are not observed in $\mathrm{TiB}_{2}{ }^{25}$ the confirmation of the topological nodal-line semimetal state by the coexistence of bulk evidence and surface signature is still desired. In this work, we investigate the electronic structure of $\mathrm{ZrB}_{2}$, which is predicted to host similar nodal-line configurations and surface states to that of $\mathrm{TiB}_{2} \cdot{ }^{26,27}$ By using ARPES and firstprinciples calculations, we clearly observe two groups of nodal rings embedded in different mirror planes. These rings are further found to be linked with each other along the $\Gamma-K$ direction. More importantly, we identify distinct surface states emanating from the bulk nodal lines, the surface signatures demonstrate the nontrivial topology of the nodal-line semimetal states in $\mathrm{ZrB}_{2}$.

Compared with the previous nodal-line candidates $\mathrm{CaAgAs}$, $\mathrm{PbTaSe}_{2}$, and the ZrSiS family, ${ }^{28-33} \mathrm{ZrB}_{2}$ has the following advantages: (1) the whole band-crossing features forming the nodal lines are clearly resolved below the Fermi energy $\left(E_{F}\right)$ in $\mathrm{ZrB}_{2}$, while the nodes of $\mathrm{CaAgAs}^{28} \mathrm{PbTaSe}_{2}{ }^{29}$ and the $\mathrm{ZrSiS}$ family $^{30-33}$ cannot be observed by ARPES with the crossings located above $E_{F ;}(2)$ the nodal-line fermions and their connections with the surface states are observed without any interference from other bands in $\mathrm{ZrB}_{2}$, while in $\mathrm{PbTaSe}_{2}{ }^{29}$ and the $\mathrm{ZrSiS}$ family, ${ }^{30-33}$

\footnotetext{
${ }^{1}$ Department of Physics and Beijing Key Laboratory of Opto-electronic Functional Materials \& Micro-nano Devices, Renmin University of China, Beijing 100872 , China; ${ }^{2}$ Shanghai Synchrotron Radiation Facility, Shanghai Institute of Applied Physics, Chinese Academy of Sciences, Shanghai 201204, China; ${ }^{3}$ State Key Laboratory of Functional Materials for Informatics and Center for Excellence in Superconducting Eletronics, SIMIT, Chinese Academy of Sciences, Shanghai 200050, China; ${ }^{4}$ National Synchrotron Radiation Laboratory, University of Science and Technology of China, Hefei 230029, China; ${ }^{5}$ Beijing National Laboratory for Condensed Matter Physics, and Institute of Physics, Chinese Academy of

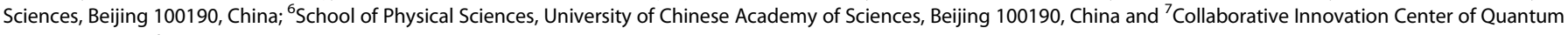
Matter, Beijing, China
}

Correspondence: Yaobo Huang (huangyaobo@sinap.ac.cn) or Hechang Lei (hlei@ruc.edu.cn) or Shancai Wang (scw@ruc.edu.cn)

These authors contributed equally: Rui Lou, Pengjie Guo, Man Li, Qi Wang.

Received: 27 March 2018 Revised: 11 August 2018 Accepted: 14 August 2018

Published online: 12 September 2018 
(a)

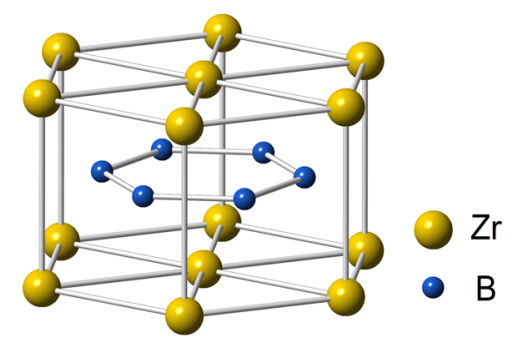

(c)

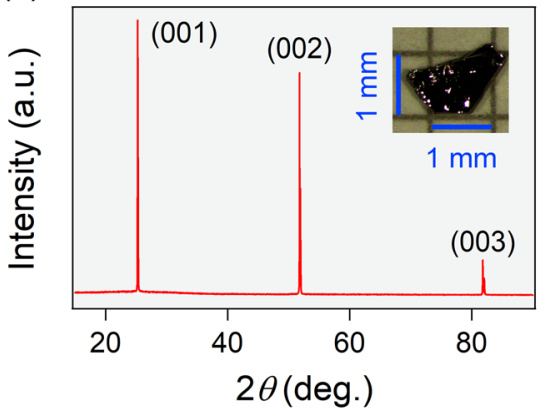

(d)

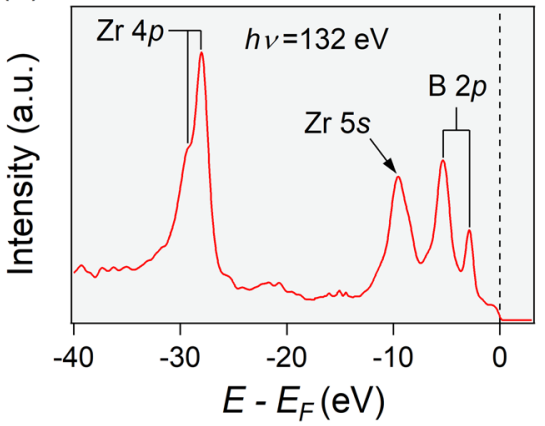

(b)

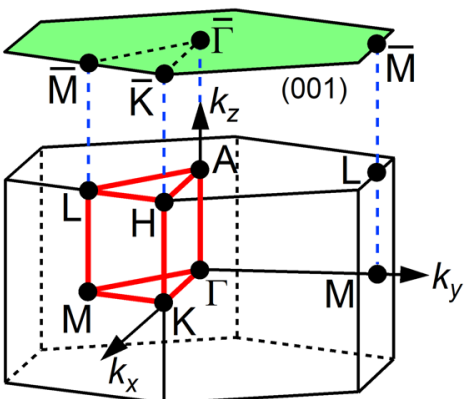

(e)

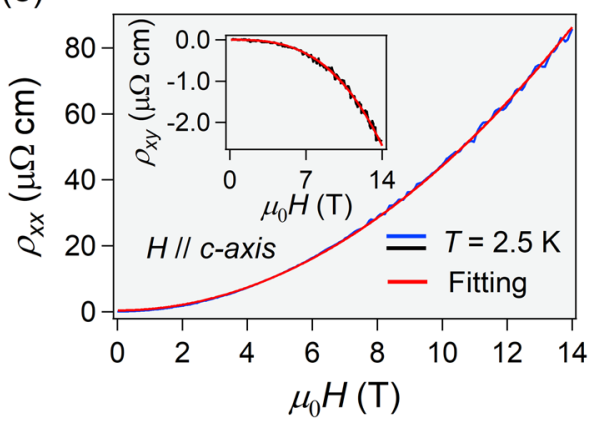

(f)

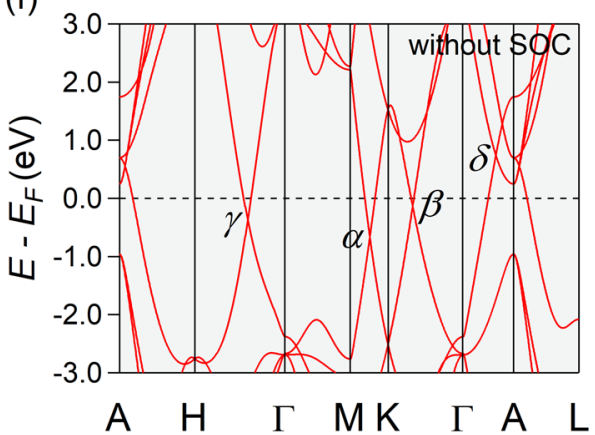

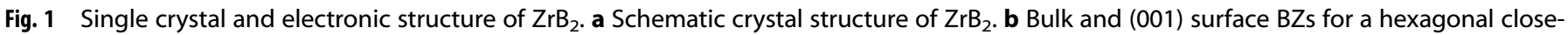

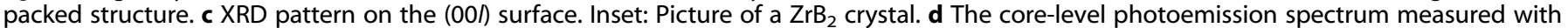

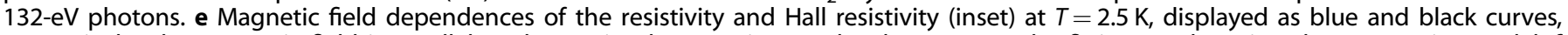

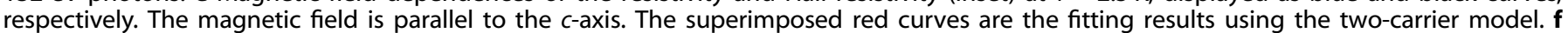

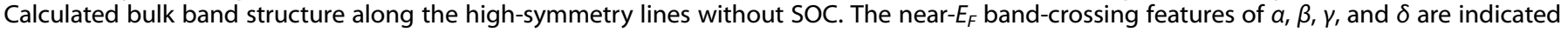

the observation of bulk nodal lines is seriously obstructed by surface states. Thus, our experimental discovery in $\mathrm{ZrB}_{2}$ establishes a unique system that has the conclusive evidence of topological nodal-line semimetal states both in the bulk and on the surface.

\section{RESULTS}

Structure and transport properties of $\mathrm{ZrB}_{2}$

As illustrated in Fig. $1 \mathrm{a}, \mathrm{ZrB}_{2}$ crystallizes in a hexagonal lattice system with the space group $P 6 / \mathrm{mmm}$ (No. 191). ${ }^{34}$ The corresponding bulk and (001)-projected surface BZs are shown in Fig. $1 \mathrm{~b}$, where the $\Gamma-K-M$ and $\Gamma-A-H$ planes are two mirror planes of $D_{6 h}$ group. The flat and shiny surface (inset of Fig. 1c) for our ARPES measurements is the $(00 /)$ plane, demonstrated by the single crystal x-ray diffraction (XRD) pattern in Fig. 1c. To further check the chemical composition of our samples, we display the core-level photoemission spectrum in Fig. 1d, where the characteristic peaks of $\mathrm{Zr}-4 p, \mathrm{Zr}-5 s$, and $\mathrm{B}-2 p$ orbitals are clearly revealed.

The magnetic field dependences of the resistivity $\left(\rho_{x x}\right)$ and Hall resistivity $\left(\rho_{x y}\right)$ at $T=2.5 \mathrm{~K}$ are depicted in Fig. $1 \mathrm{e}$ and the inset of
Fig. 1e, respectively. By fitting the experimental curves with the two-carrier model, ${ }^{35}$ the estimated densities of electron-type and hole-type carriers are $1.156(5) \times 10^{21}$ and $1.153(5) \times 10^{21} \mathrm{~cm}^{-3}$, respectively, comparable to that of $\mathrm{TiB}_{2}\left(n_{e}, n_{h} \sim 10^{21} \mathrm{~cm}^{-3}\right)^{25}$ and the ZrSiS family $\left(n_{e}, n_{h} \sim 10^{20} \mathrm{~cm}^{-3}\right) .^{36,37}$ Figure if presents an overview of the calculated bulk band structure for $\mathrm{ZrB}_{2}$ without the spin-orbit coupling (SOC) effect. Four Dirac-like band-crossing features, which are denoted as $a, \beta, \gamma$, and $\delta$, respectively, give rise to two groups of nodal rings embedded in different mirror planes. When the SOC is included in the calculation, small energy gaps ( $40 \mathrm{meV})$ open at these band crossings. ${ }^{38}$ We will proceed to a detailed discussion on the nodal-line semimetal states by systematic electronic structure investigations in the following.

Fermi surface (FS) topologies in four high-symmetry planes We present the measured and calculated FS topologies in Fig. 2. Figure $2 \mathrm{~d}-\mathrm{f}$ shows the FSs recorded with three different photon energies, $h v=132,94$, and $70 \mathrm{eV}$, which are close to the $k_{z} \sim \pi$ (FS3), $\sim 0$ (FS1), and $\sim \pi$ (FS2) planes, respectively. By continuously varying the photon energy, we are able to map out the FS in the $\Gamma$ $A-H$ plane (FS4), as displayed in Fig. $2 \mathrm{~b}$. From the FS1 in the $k_{z} \sim 0$ 
(a)

(d)

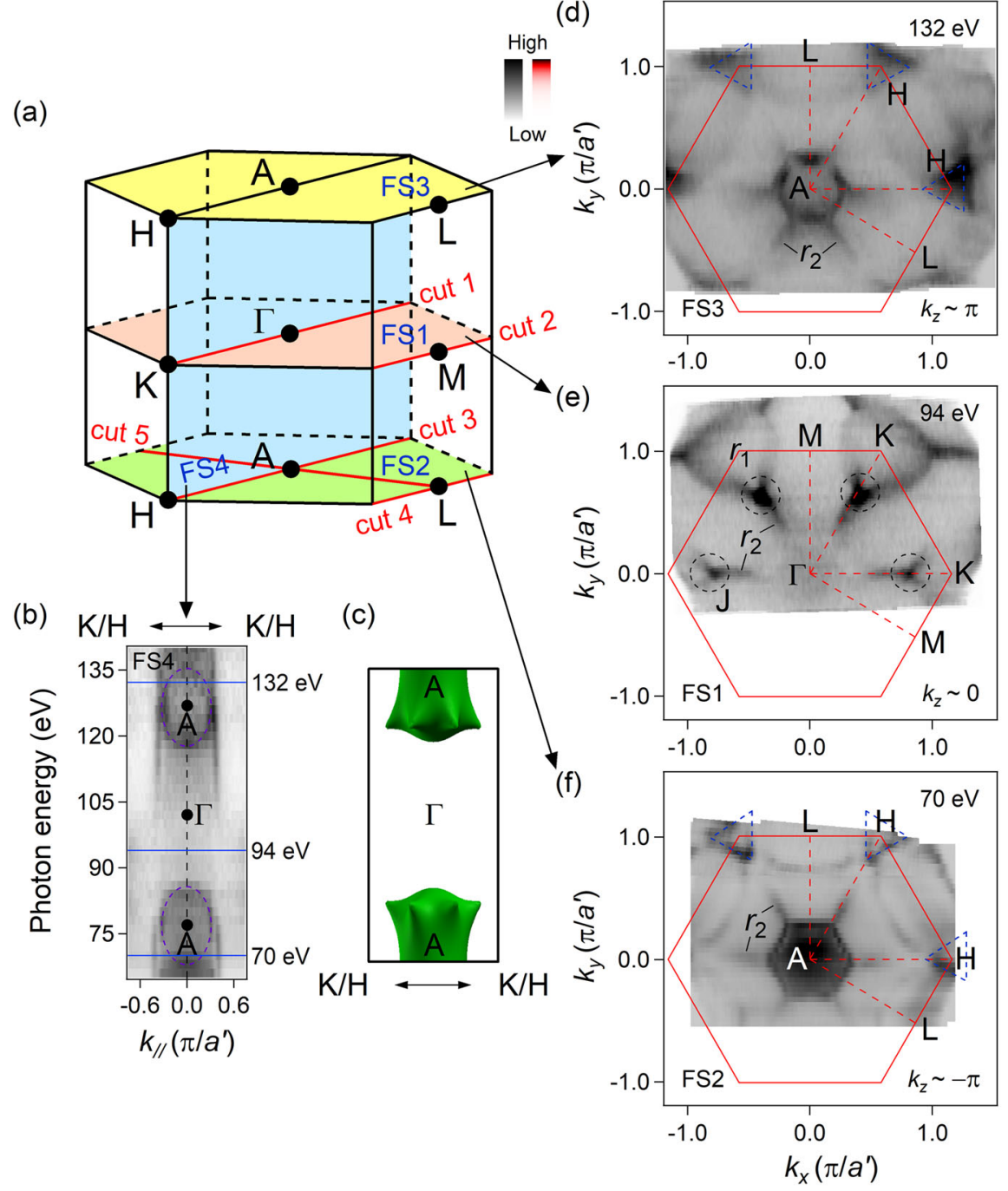

(g)

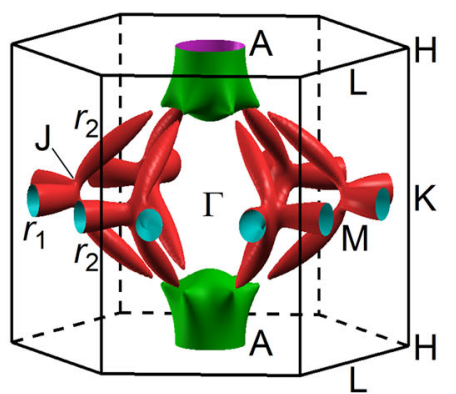

(h)

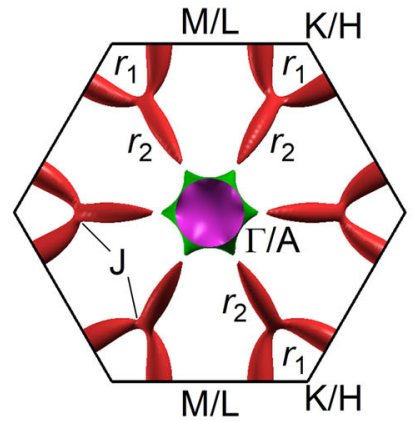

(i)

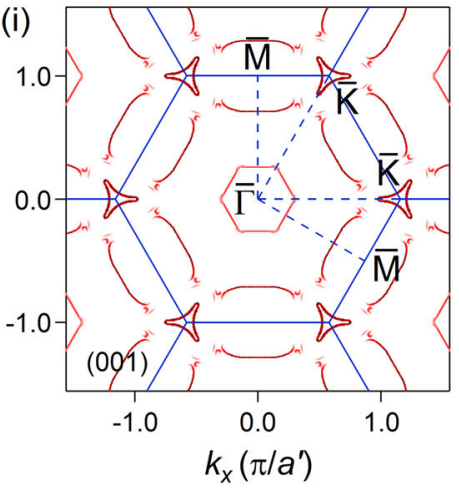

Fig. 2 Experimental and theoretical FSs of $\mathrm{ZrB}_{2}$. a 3D bulk BZ with four high-symmetry planes, which illustrate the momentum locations of the measured FSs in (b, d-f). Cuts 1, 2 and 3-5 indicate the locations of the experimental band structures in Figs. 3 and 4, respectively. $\mathbf{b}$ ARPES intensity map [FS4 in (a)] at $E_{F}$ in the $h v-k_{\|}$plane, where $k_{\|}$is oriented along the $\Gamma-K(A-H)$ direction, recorded with various photon energies. $a^{\prime}$ $=\sqrt{3} a / 2(a=3.169 \AA)$. The purple dashed ellipses indicate the hole-like FSs at $A$ points, serving as guides to the eye. $\mathbf{c}$ Projections of calculated $3 D$ FSs on the $\Gamma-A-H$ plane. d-f ARPES intensity plots obtained by $h v=132,94$, and 70 eV, showing FS3, FS1, and FS2 indicated in (a), respectively. The red solid lines indicate the first BZs projected on the (001) surfaces. Two groups of Dirac nodal rings, $r_{1}$ and $r_{2}[(001)$ projection], are resolved. The link points (denoted as $J$ point) of $r_{1}$ and $r_{2}$ are indicated by black dashed circles in (e). The blue dashed triangles in $(\mathbf{d}, \mathbf{f})$ are guides to the eye for the triangular FSs around $H$ points. $\mathbf{g}$ Calculated 3D bulk FSs. $\mathbf{h}$ The top view of the calculated 3D FSs, namely the two-dimensional FSs projected on the (001) surface. i Calculated FS contours of the (001) surface for a 20-unit-cell-thick slab with Zr terminations. The intensity of the red color scales the spectral weight projected to the topmost unit cell. The blue solid lines represent the (001) surface BZs

plane (Fig. 2e), the $r_{1}$ nodal rings formed by $a$ and $\beta$ are clearly observed surrounding $K$ points. From the FS2 and FS3 in the $k_{z} \sim$ $\pm \pi$ planes (Fig. $2 d, f$ ), triangular FSs are resolved at $H$ points, which are attributed as the surface states and discussed later, but no sign of the $r_{1}$ nodal ring is revealed. Another major contrast between FS1 and FS2/FS3 is the absence of intensity around $\Gamma$ point for the former, and the presence of a circular FS centered at $A$ point for the latter. This difference could be further illuminated by the FS4 in the $\Gamma-A-H$ plane (Fig. 2b). The FS around $A$ point, which agreeing well with the green-colored FS sheet in the calculation (Fig. 2c, g), arises from a hole-like band presented in Fig. 4. Characterized by these features in experiment, the electronic structure exhibits a prominent three-dimensional (3D) nature, as confirmed by the calculated 3D FSs (Fig. $2 \mathrm{~g}$ ) and their projections on the $\Gamma-A-H$ plane (Fig. 2c). Since the $r_{2}$ nodal ring in the $\Gamma-A-H$ plane formed by $\gamma$ and $\delta$ also pass through the Fermi wave vectors of $\beta$, thereby the $\beta$ band-crossing feature belongs to both the $r_{1}$ and $r_{2}$ nodal rings. Consequently, the 3D FS is a nodal-link structure composed of these two nodal rings. Due to the $k_{z}$ broadening effect, which is prominent in ARPES measurements with vacuum ultraviolet light, $31,39,40$ the ARPES spectra reflects the electronic states integrated over a certain $k_{z}$ region of bulk BZ. Therefore, the (001)-projected $r_{2}$ nodal rings around $\Gamma / A$ points are clearly resolved in FS1-FS3 (Fig. 2d-f). Furthermore, the nodal-link point $(J)$ of the $r_{1}$ and $r_{2}$ nodal rings along the $\Gamma-K$ direction is unambiguously recognized in FS1 (Fig. 2e). All the above observations are consistent with the calculated bulk FSs shown in Fig. 2c, $g, h$.

In addition, we observe that some FS features in the $k_{z} \sim \pm \pi$ planes (FS2 and FS3) are absent in the calculated bulk FSs, i.e., the triangular FSs around $H$ points, the FS sheets between two $H$ points, and the hexagonal FS centered at $A$ point (relatively weak 
(a)

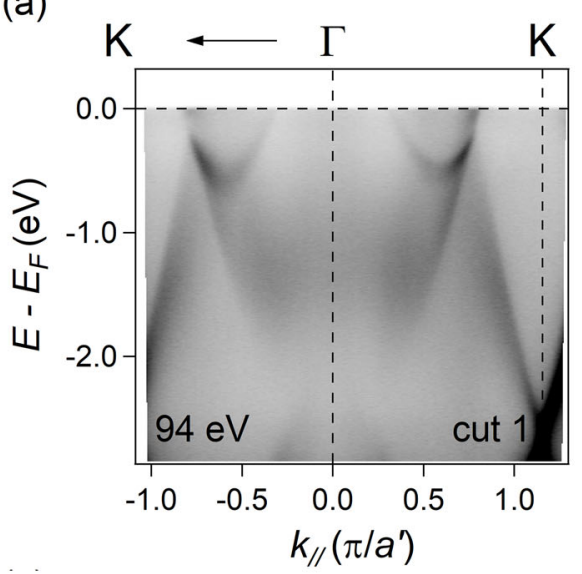

(c)

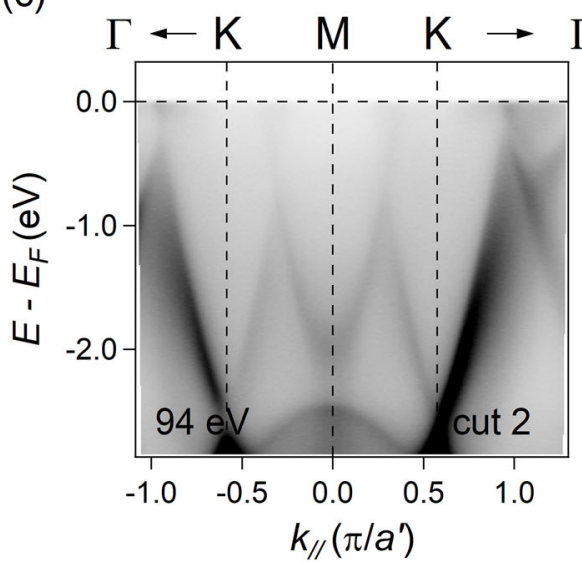

(b)

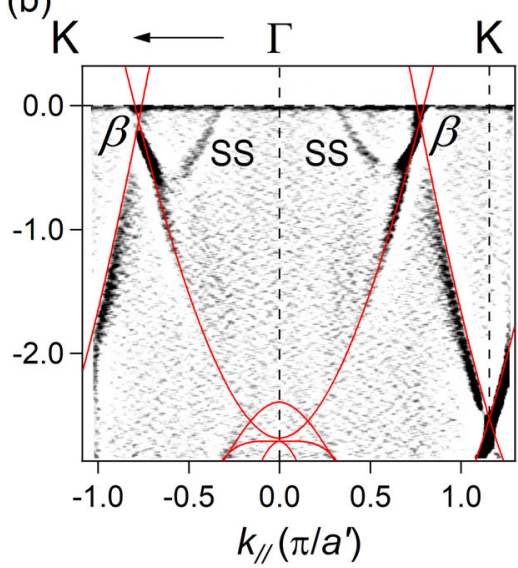

(d)

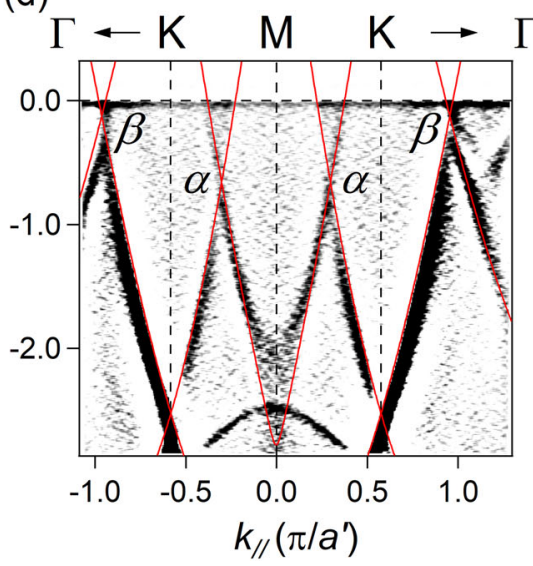

(e)

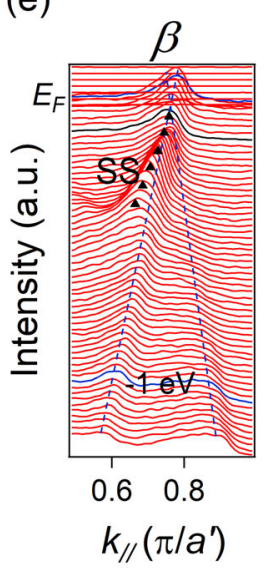

(f)

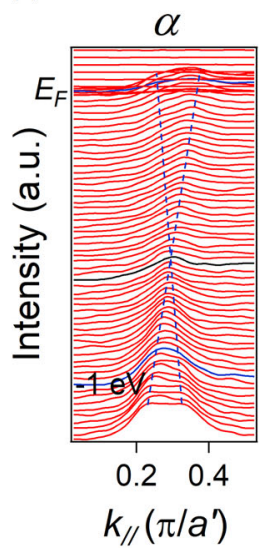

Fig. 3 Band structure of the $r_{1}$ nodal ring. $\mathbf{a}, \mathbf{b}$ Photoemission intensity plot and corresponding second derivative plot along the $\Gamma-K$ direction (cut 1 in Fig. 2a) recorded with $h v=94 \mathrm{eV}$, respectively, where SS is short for surface state. (c, d) Same as (a, b), respectively, but along the $M-K$ direction (cut 2 in Fig. 2a). The appended red curves in (b, d) are calculated bulk band dispersions without SOC. The band-crossing features along the $M-K$ and $\Gamma-K$ directions are denoted as $a$ and $\beta$, respectively. e, $\mathbf{f}$ MDC plots of $\beta$ and $a$, which are taken around the right ones in (a, $\mathbf{c}$ ), respectively. The blue dashed lines indicate the linear dispersions. The band-crossing points are highlighted by the black curves. The solid triangles in (e) are extracted peak positions, serving as guides to the eye for the surface state emanating from the node of $\beta$

in FS3 due to the matrix element effect and its presence can be proved in Fig. 2b). As illustrated in the $h v-k_{\|}\left[k_{\|}\right.$is oriented along the $\Gamma-K(A-H)$ direction] ARPES intensity plot in Fig. $2 b$, the Fermi crossings of the hexagonal FS do not show noticeable photonenergy dependence over a wide range (65-140 eV), which is an indication of surface state. This result inspires us to carry out surface state spectrum calculations for a (001)-oriented 20-unitcell-thick slab terminated by Zr layers. The calculated surface state FSs presented in Fig. $2 \mathrm{i}$ well reproduce these three experimental FS topologies, demonstrating their surface state origins.

Presence of bulk nodal-line fermions below $E_{F}$

Based on the discussion above, the $r_{1}$ nodal rings can only be observed in the $k_{z} \sim 0$ plane. This character establishes an excellent system to separately investigate the bulk nodal lines and electronic surface states. Now we record the near- $E_{F}$ ARPES spectra along the $\Gamma-K$ and $M-K$ directions, which are indicated as cuts 1 and 2 in Fig. $2 \mathrm{a}$, respectively, in the $k_{z} \sim 0$ plane (FS1) to prove the presence of bulk nodal-line fermions in $\mathrm{ZrB}_{2}$. The intensity plots and corresponding second derivative plots are shown in Fig. 3a-d. The electronic structure near $E_{F}$ is only composed of the $a$ and $\beta$ band-crossing features forming the $r_{1}$ nodal rings, except a band dispersing from the crossing point of $\beta$ along the $\Gamma-K$ direction, which is relatively weak when crossing $E_{F}$ due to the matrix element effect and thus not resolved in the FS mapping data in Fig. 2e. This band is identified as the surface state and discussed later. We then plot the momentum distribution curves (MDCs) of $\beta$ and $a$ in Fig. 3e, $f$, respectively. The linear dispersions in a large energy range with the crossing points below $E_{F}$ are unambiguously recognized. By comparing with the superimposed bulk band calculations in Fig. 3b, d one can obtain a high consistency between experiment and theory, which provides further evidence for the experimental realization of bulk nodal-line fermions in $\mathrm{ZrB}_{2}$. As for the hole-like band $\sim 2.5 \mathrm{eV}$ below $E_{F}$ at $M$ point (Fig. $3 c, d$ ), due to the $k_{z}$ broadening effect in ARPES, it derives from the projection of the band along the $H-L-H$ direction with a similar feature in Fig. $4 \mathrm{c}$, which is well reproduced by the calculation.

Electronic surface states associated with the bulk nodal lines Next, from the perspective of surface signature, we demonstrate the nontrivial topology of the nodal-line semimetal states in $\mathrm{ZrB}_{2}$ by investigating the surface states revealed in the $k_{z} \sim-\pi$ plane (FS2). In Fig. 4a, c, e, we present the experimental band dispersions with overlapped bulk band calculations along the $A-H, L-H$, and $A-L$ directions, as indicated by cuts $3-5$ in Fig. 2a, respectively. Besides the well-reproduced bulk bands, there are some extra bands (SS1-SS3) not existing in the bulk calculations. According to the experimental FSs measured in the $k_{z} \sim \pm \pi$ planes (FS2 and FS3), we can determine the origins of the crossing- $E_{F}$ ones among these 
(a)

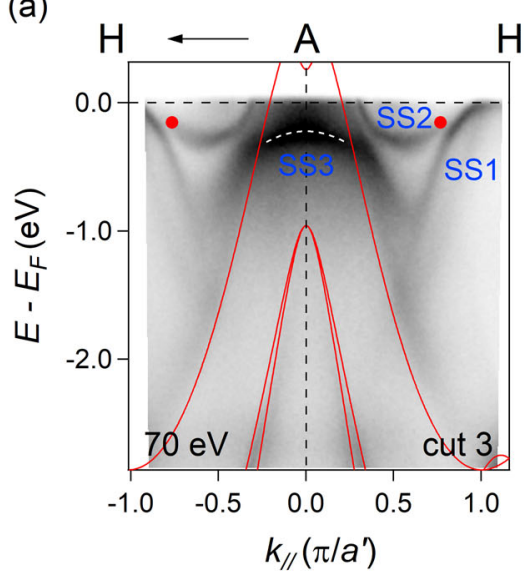

(b)

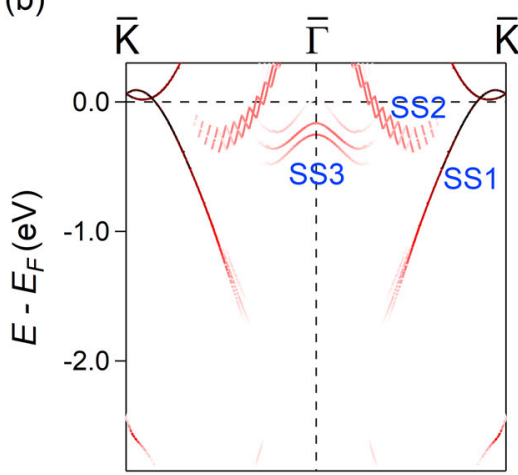

(c)

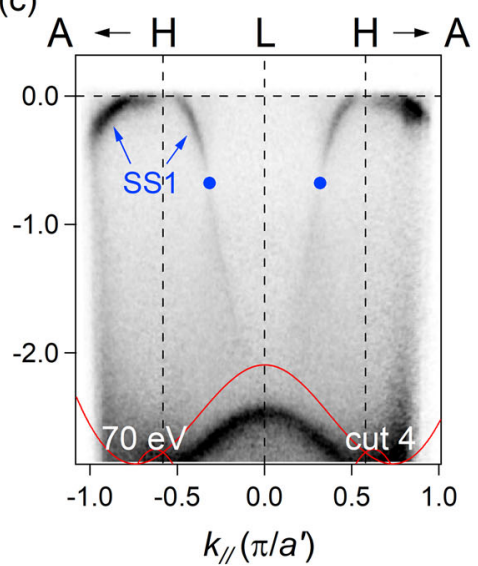

(d)

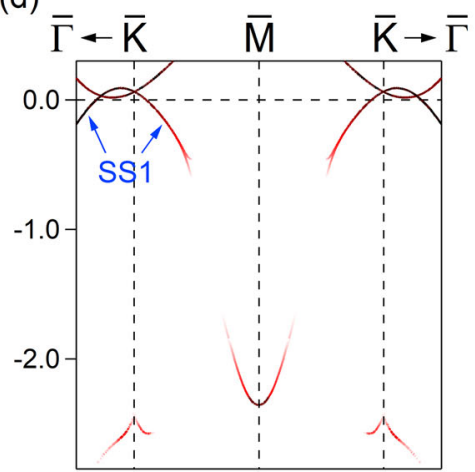

(e)

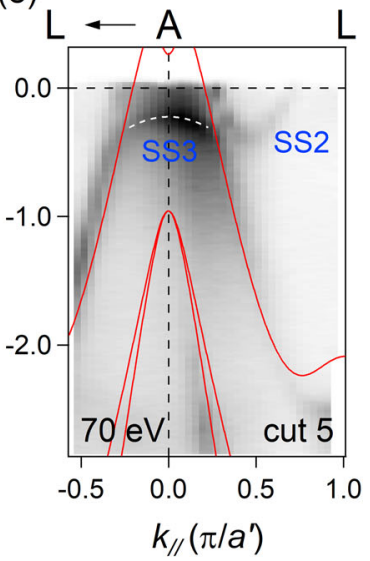

(f)

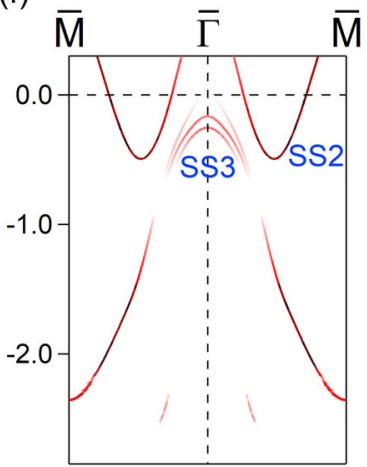

Fig. 4 Electronic surface states in the $k_{z} \sim-\pi$ plane. a, c, e Photoemission intensity plots along the $A-H, L-H$, and $A-L$ directions (cuts $3-5$ in Fig. 2a) measured with $h v=70 \mathrm{eV}$, respectively. The appended red curves are calculated bulk band dispersions without SOC. The white dashed curves in $(\mathbf{a}, \mathbf{e})$ indicate the SS3 at $A$ point, serving as guides to the eye. The red and blue solid circles in (a, $\mathbf{c})$ represent the crossing points of $\beta$ and $a$, whose positions are determined by the MDCs in Fig. 3e, f, respectively. b, d, f Calculated band structure along the $\bar{\Gamma}-\bar{K}, \bar{M}-\bar{K}$, and $\bar{\Gamma}-\bar{M}$ directions for a 20-unit-cell-thick (001) slab with Zr terminations, respectively. The intensity of the red color scales the spectral weight projected to the topmost unit cell

bands, i.e., the SS1 corresponds to the triangular FS around $H$ point, and the SS2 forms the hexagonal FS surrounding $A$ point and the FS sheet between two $H$ points, respectively. To further understand the three extra bands, we perform 20-unit-cell-thick slab model calculations with Zr-terminated layers. The calculated band dispersions along the $\bar{\Gamma}-\bar{K}, \bar{M}-\bar{K}$, and $\bar{\Gamma}-\bar{M}$ directions with spectral weight from the topmost unit cell are plotted in Fig. $4 b, d$, $\mathrm{f}$, respectively, which can reproduce SS1-SS3 very well and confirm their surface state nature.

\section{DISCUSSION}

In order to clarify the nontrivial topology of the bulk nodal lines realized in $\mathrm{ZrB}_{2}$, which are protected by the mirror-reflection symmetries and the combination of spatial-inversion symmetry $(P)$ and time-reversal symmetry $(T)$, i.e., the $P \cdot T$ symmetry, here we illustrate the connection between the nodal lines and the surface states by combining both the bulk and surface observations. We plot the crossing points of $\beta$ and $a$ as the red and blue solid circles in Fig. $4 a$, c, whose positions are extracted from the MDCs in Fig. $3 e, f$, respectively. We can observe that the bulk nodes of $\beta$ and $a$ exactly locate on the loci of the surface states resolved in the $k_{z} \sim$ $-\pi$ plane. Along the $A-H$ direction (Fig. 4a), starting from the node of $\beta$, the SS2 disperses inwards with respect to $A$ point. This behavior resembles that of the extra band observed along the $\Gamma-K$ direction in Fig. 3, showing its (the extra band around $\Gamma$ point) surface state origin associated with the bulk nodal line. Along the $L-H$ direction (Fig. 4C), the SS1 passing through the node of $a$ disperses outwards with respect to $L$ point. The bulk nodes and the surface state spectra are obtained from two independent measurements carried out in different high-symmetry planes, therefore the discovery of their well match provides convincing evidence for the topological nature (the bulk-boundary correspondence ${ }^{8,22,41}$ ) of the nodal-line semimetal states in $\mathrm{ZrB}_{2}$.

In summary, our direct experimental observation by ARPES presents conclusive evidence of the bulk nodal-line fermions in $\mathrm{ZrB}_{2}$ with negligible SOC effect. Under the protection of the mirror-reflection symmetries and the P.T symmetry, the electronic structure hosts two groups of Dirac nodal rings, which are linked together along the $\Gamma-K$ direction. Meanwhile, we clearly resolve electronic surface state emanating from each nodal line, proving the nontrivial topology of the bulk nodal lines. With the experimental realization of topological nodal-line semimetal states in $\mathrm{ZrB}_{2}$ powerfully supported by both the bulk evidence and the surface signature, we establish an ideal material platform for studying the novel physics and exotic properties related to nodal lines.

\section{METHODS}

Sample synthesis

High-quality single crystals of $\mathrm{ZrB}_{2}$ were grown via the Fe flux method. ${ }^{38}$ The starting elements of $\mathrm{Zr}$ (99.95\%), B (99.99\%), and Fe (99.98\%) were put into an alumina crucible, with a molar ratio of $\mathrm{Zr}: \mathrm{B}: \mathrm{Fe}=3: 6: 17$. The mixture was heated up to $1873 \mathrm{~K}$ in a high-purity argon atmosphere and then 
slowly cooled down to $1623 \mathrm{~K}$ at a rate of $4 \mathrm{~K} / \mathrm{h}$. The $\mathrm{ZrB}_{2}$ single crystals were separated from the Fe flux using the hot hydrochloric acid solution.

\section{ARPES measurements}

ARPES measurements were performed at the Dreamline beamline of the Shanghai Synchrotron Radiation Facility using a Scienta D80 analyzer and at the beamline $13 \mathrm{U}$ of the National Synchrotron Radiation Laboratory equipped with a Scienta R4000 analyzer. The energy and angular resolutions were set to $25 \mathrm{meV}$ and $0.2^{\circ}$, respectively. Fresh surfaces for ARPES measurements were obtained by cleaving the samples in situ along the (001) plane. All spectra presented in this work were recorded at $T=$ $20 \mathrm{~K}$ in a working vacuum better than $5 \times 10^{-11}$ Torr.

\section{Band structure calculations}

First-principles calculations were carried out with the projector augmented-wave method ${ }^{42,43}$ as implemented in the Vienna $a b$ initio Simulation Package. ${ }^{44}$ The generalized gradient approximation of PerdewBurke-Ernzerhof formula ${ }^{45}$ was adopted for the exchange-correlation functional. The kinetic energy cutoff of the plane-wave basis was set to be $420 \mathrm{eV}$. A $20 \times 20 \times 20 k$-point mesh was utilized for the BZ sampling. The bulk FSs were investigated by adopting the maximally localized Wannier function method. ${ }^{46} \mathrm{~A}$ slab with thickness of 20 unit cells along the [001] direction was used in our surface state calculations. The slabs were separated by a vacuum layer of $20 \AA$, which was sufficient to avoid the interactions between different slabs. SOC effect ${ }^{38}$ was not taken into account in all the above calculations.

\section{DATA AVAILABILITY}

All relevant data are available from the corresponding authors upon request.

\section{ACKNOWLEDGEMENTS}

The work was supported by the National Key R\&D Program of China (Grants No. 2016YFA0300504 and No. 2017YFA0302903), the National Natural Science Foundation of China (Grants No. 11774421, No. 11574394, No. 11774423, No. 11774424, No. 11227902, and No. 11704394), and the Chinese Academy of Sciences (CAS) (Project No. XDB07000000). R.L., K.L., and H.L. were supported by the Fundamental Research Funds for the Central Universities, and the Research Funds of Renmin University of China (RUC) (Grants No. 17XNH055, No. 14XNLQ03, No. 15XNLF06, and No. 15XNLQ07). Y.H. was supported by the CAS Pioneer Hundred Talents Program. Z.L. acknowledges Shanghai Sailing Program (No. 17YF1422900).

\section{AUTHOR CONTRIBUTIONS}

R.L., H.C.L., Y.B.H., H.D., and S.C.W. conceived the experiments. R.L. performed ARPES measurements with the assistance of M.L., Z.H.L., X.C.W., Z.L.W., Z.S., and D.W.S. P.J.G., K.L., and Z.Y.L. performed first-principles calculations. Q.W., S.S.S., C.H.L., and H.C.L. synthesized the single crystals. R.L., H.C.L., and S.C.W. analysed the experimental data. R.L. plotted the figures. R.L. and S.C.W. wrote the manuscript.

\section{ADDITIONAL INFORMATION}

Supplementary information accompanies the paper on the npj Quantum Materials website (https://doi.org/10.1038/s41535-018-0121-4).

Competing interests: The authors declare no competing interests.

Publisher's note: Springer Nature remains neutral with regard to jurisdictional claims in published maps and institutional affiliations.

\section{REFERENCES}

1. Hasan, M. Z. \& Kane, C. L. Colloquium: topological insulators. Rev. Mod. Phys. 82, 3045 (2010).

2. Qi, X.-L. \& Zhang, S.-C. Topological insulators and superconductors. Rev. Mod. Phys. 83, 1057 (2011).

3. Weng, H., Dai, X. \& Fang, Z. Exploration and prediction of topological electronic materials based on first-principles calculations. MRS Bull. 39, 849-858 (2014).

4. Weng, H., Dai, X. \& Fang, Z. Topological semimetals predicted from first-principles calculations. J. Phys. Condens. Matter 28, 303001 (2016).
5. Volovik, G. E. The Universe in a Helium Droplet. (Oxford University Press, Oxford, 2009).

6. Fang, Z. et al. The anomalous Hall effect and magnetic monopoles in momentum space. Science 302, 92-95 (2003).

7. Ryu, S. \& Hatsugai, Y. Topological origin of zero-energy edge states in particlehole symmetric systems. Phys. Rev. Lett. 89, 077002 (2002).

8. Fang, C., Weng, H. M., Dai, X. \& Fang, Z. Topological nodal line semimetals. Chin. Phys. B 25, 117106 (2016).

9. Li, R. et al. Dirac node lines in pure alkali earth metals. Phys. Rev. Lett. 117, 096401 (2016).

10. Wan, X., Turner, A. M., Vishwanath, A. \& Savrasov, S. Y. Topological semimetal and Fermi-arc surface states in the electronic structure of pyrochlore iridates. Phys. Rev. B 83, 205101 (2011).

11. Wang, Z. et al. Dirac semimetal and topological phase transitions in $A_{3} \mathrm{Bi}(A=\mathrm{Na}$, K, Rb). Phys. Rev. B 85, 195320 (2012).

12. Weng, H., Fang, C., Fang, Z., Bernevig, B. A. \& Dai, X. Weyl semimetal phase in noncentrosymmetric transition-metal monophosphides. Phys. Rev. X 5, 011029 (2015).

13. Huang, S.-M. et al. A Weyl Fermion semimetal with surface Fermi arcs in the transition metal monopnictide TaAs class. Nat. Commun. 6, 7373 (2015).

14. Soluyanov, A. A., Gresch, D., Wang, Z., Wu, Q. S. \& Troyer, M. Type-II Weyl semimetals. Nature 527, 495-498 (2015).

15. Chang, G. et al. Universal topological electronic properties of nonmagnetic chiral crystals. arXiv 1611.07925 (2016).

16. Liu, Z. K. et al. Discovery of a three-dimensional topological Dirac semimetal, $\mathrm{Na}_{3} \mathrm{Bi}$. Science 343, 864-867 (2014).

17. Borisenko, S. et al. Experimental realization of a three-dimensional Dirac semimetal. Phys. Rev. Lett. 113, 027603 (2014).

18. Lv, B. Q. et al. Experimental discovery of Weyl semimetal TaAs. Phys. Rev. X 5, 031013 (2015).

19. Xu, S.-Y. et al. Discovery of a Weyl fermion semimetal and topological Fermi arcs. Science 349, 613-617 (2015).

20. Lv, B. Q. et al. Observation of Weyl nodes in TaAs. Nat. Phys. 11, 724-727 (2015).

21. Yang, L. X. et al. Weyl semimetal phase in the non-centrosymmetric compound TaAs. Nat. Phys. 11, 728-732 (2015).

22. Burkov, A. A., Hook, M. D. \& Balents, L. Topological nodal semimetals. Phys. Rev. $B$ 84, 235126 (2011).

23. Yang, S.-Y. et al. Symmetry demanded topological nodal-line materials. Adv. Phys.: $X$ 3, 1414631 (2018).

24. Yu, R., Fang, Z., Dai, X. \& Weng, H. Topological nodal line semimetals predicted from first-principles calculations. Front. Phys. 12, 127202 (2017).

25. Liu, Z.-H. et al. Experimental observation of Dirac nodal links in centrosymmetric semimetal $\mathrm{TiB}_{2}$. Phys. Rev. X, 8, 031044 (2018).

26. Zhang, X., Yu, Z. M., Sheng, X. L., Yang, H. Y. \& Yang, S. A. Coexistence of four-band nodal rings and triply degenerate nodal points in centrosymmetric metal diborides. Phys. Rev. B 95, 235116 (2017).

27. Feng, X., Yue, C., Song, Z., Wu, Q. \& Wen, B. Topological Dirac nodal-net fermions in $\mathrm{AlB}_{2}$-type $\mathrm{TiB}_{2}$ and $\mathrm{ZrB}_{2}$. Phys. Rev. Mater. 2, 014202 (2018).

28. Wang, X.-B. et al. Topological surface electronic states in candidate nodal-line semimetal CaAgAs. Phys. Rev. B 96, 161112(R) (2017).

29. Bian, G. et al. Topological nodal-line fermions in spin-orbit metal PbTaSe ${ }_{2}$. Nat. Commun. 7, 10556 (2016).

30. Schoop, L. M. et al. Dirac cone protected by non-symmorphic symmetry and three-dimensional Dirac line node in ZrSiS. Nat. Commun. 7, 11696 (2016).

31. Takane, D. et al. Dirac-node arc in the topological line-node semimetal HfSiS. Phys. Rev. B 94, 121108(R) (2016).

32. Lou, R. et al. Emergence of topological bands on the surface of ZrSnTe crystal. Phys. Rev. B 93, 241104(R) (2016).

33. Hosen, $M$. M. et al. Tunability of the topological nodal-line semimetal phase in ZrSiX-type materials (X = S, Se, Te). Phys. Rev. B 95, 161101(R) (2017).

34. Post, B., Glaser, F. W. \& Moskowitz, D. Transition metal diborides. Acta Metall. 2, 20-25 (1954).

35. Xia, B. et al. Indications of surface-dominated transport in single crystalline nanoflake devices of topological insulator $\mathrm{Bi}_{1.5} \mathrm{Sb}_{0.5} \mathrm{Te}_{1.8} \mathrm{Se}_{1.2}$. Phys. Rev. B 87, 085442 (2013).

36. $\mathrm{Hu}$, J. et al. Evidence of topological nodal-line fermions in ZrSiSe and ZrSiTe. Phys. Rev. Lett. 117, 016602 (2016).

37. $\mathrm{Hu}$, J. et al. Nearly massless Dirac fermions and strong Zeeman splitting in the nodal-line semimetal ZrSiS probed by de Haas-van Alphen quantum oscillations. Phys. Rev. B 96, 045127 (2017).

38. Wang, Q. et al. Extremely large magnetoresistance and high-density Dirac-like fermions in $\mathrm{ZrB}_{2}$. Phys. Rev. B 97, 205105 (2018).

39. Strocov, V. N. Intrinsic accuracy in 3-dimensional photoemission band mapping. J. Electron Spectrosc. Relat. Phenom. 130, 65-78 (2003). 
40. Kumigashira, H. et al. High-resolution angle-resolved photoemission study of LaSb. Phys. Rev. B 58, 7675 (1998).

41. Weng, H. et al. Topological node-line semimetal in three-dimensional graphene networks. Phys. Rev. B 92, 045108 (2015).

42. Blöchl, P. E. Projector augmented-wave method. Phys. Rev. B 50, 17953 (1994).

43. Kresse, G. \& Joubert, D. From ultrasoft pseudopotentials to the projector augmented-wave method. Phys. Rev. B 59, 1758 (1999).

44. Kresse, G. \& Hafner, J. Ab initio molecular dynamics for open-shell transition metals. Phys. Rev. B 48, 13115 (1993).

45. Perdew, J. P., Burke, K. \& Ernzerhof, M. Generalized gradient approximation made simple. Phys. Rev. Lett. 77, 3865 (1996).

46. Souza, I., Marzari, N. \& Vanderbilt, D. Maximally localized Wannier functions for entangled energy bands. Phys. Rev. B 65, 035109 (2001).
Open Access This article is licensed under a Creative Commons Attribution 4.0 International License, which permits use, sharing, adaptation, distribution and reproduction in any medium or format, as long as you give appropriate credit to the original author(s) and the source, provide a link to the Creative Commons license, and indicate if changes were made. The images or other third party material in this article are included in the article's Creative Commons license, unless indicated otherwise in a credit line to the material. If material is not included in the article's Creative Commons license and your intended use is not permitted by statutory regulation or exceeds the permitted use, you will need to obtain permission directly from the copyright holder. To view a copy of this license, visit http://creativecommons. org/licenses/by/4.0/.

(c) The Author(s) 2018 